\title{
Constraints on anisotropic RG flows from the holographic entanglement entropy
}

\author{
Mostafa Ghasemi ${ }^{1, *}$ and Shahrokh Parvizi $\odot^{2, \dagger}$ \\ ${ }^{1}$ School of Particles and Accelerators, Institute for Research in Fundamental Sciences (IPM) \\ P.O. Box 19395-5531, Tehran, Iran \\ ${ }^{2}$ Department of Physics, School of Sciences, Tarbiat Modares University, \\ P.O. Box 14155-4838, Tehran, Iran
}

(Received 11 July 2021; accepted 13 September 2021; published 22 October 2021)

\begin{abstract}
In the context of the gauge/gravity duality, using the proposed candidate $c$-function, which is derived from the entanglement entropy of a strip-shaped region, we investigate the RG flow for $d+1$-dimensional quantum field theories with broken Lorentz and rotational symmetries in the IR, but preserved conformal invariance in the UV boundary. We examine conditions of monotonicity of the $c$-function for holographic anisotropic theories dual to the Einstein gravity via the constraints imposed by the null energy conditions. We consider near UV and IR behaviors and identify the sufficient conditions that guarantee the $c$ function decreases monotonically along the RG flows.
\end{abstract}

DOI: 10.1103/PhysRevD.104.086028

\section{INTRODUCTION}

A challenging question in the quantum field theory (QFT) is finding a proper quantity in order to probe the structure of parameter space of QFT. The renormalization group (RG) provides a framework to study various aspects of the QFT space. The RG denotes how physics in different energy scales can be related to each others.

There are special points in this space, called fixed points, which correspond to theories with scaling symmetry. Other theories may be derived from such points by various deformations. By definition, this quantity/function is definite positive and monotonically decreases along the RG flows and is a measure of degrees of freedom of the effective theory. Another feature of this function is that its values at fixed points equal to the central charges of the corresponding theories.

In the space of two-dimensional QFT, Zamolodchikov [1] showed that there exists such a positive definite function $c_{2}$, which decreases monotonically along the RG flows. This function is stationary and coincides with the central charge $c$ of the associated fixed points CFT. As a consequence, if RG flows connect two UV and IR fixed points, we have

\footnotetext{
*ghasemi.mg@ipm.ir

parvizi@modares.ac.ir
}

Published by the American Physical Society under the terms of the Creative Commons Attribution 4.0 International license. Further distribution of this work must maintain attribution to the author(s) and the published article's title, journal citation, and DOI. Funded by SCOAP ${ }^{3}$.

$$
c_{\mathrm{UV}}>c_{\mathrm{IR}}
$$

From the Wilsonian point of view, it is a measure of the effective degrees of freedom that decreases along the RG flows.

One of the suitable candidates $c$-function is emergent in the realm of the information theory. In this context, the proper $c$-function is derived from the entanglement entropy that is a measure of quantum entanglement. It can be used as a measure of effective degrees of freedom in QFT along the RG flows in the Wilsonian sense. For a twodimensional conformal field theory, the entanglement entropy for an interval of length $l$ is given by $[2,3]$

$$
S_{\mathrm{EE}}^{\mathrm{CFT}}=\frac{c}{3} \log \left(\frac{l}{\delta}\right)+\cdots
$$

where $c$ is the central charge, $\delta$ is a UV regulator, and dots denote the $l$-independent nonuniversal terms.

The authors $[4,5]$ reformulated the Zamolodchikov's c-theorem for a two-dimensional QFT in terms of $S_{\mathrm{EE}}$ and defined

$$
c_{2}=3 l \frac{d S_{\mathrm{EE}}(l)}{d l}
$$

The monotonicity flow of the $c_{2}, d c_{2} / d l \leq 0$, can be shown from the strong subadditivity property of $S_{\mathrm{EE}}$ as well as the Lorentz symmetry and unitarity of the underlying QFT. It takes the value $c_{2}=c$ at $\mathrm{RG}$ fixed points.

The generalization of the $c$-theorem for higher dimensions is remained a challenging problem. This issue has 
been addressed in [6-9] for three- and four-dimensional theories with Lorentz invariance. On the other hand, the gauge/gravity duality provides a framework to investigate the RG flows to arbitrary dimensions in the gravitational context [10-14]. The connection between RG flows and the entanglement entropy well is depicted via holographic prescription of computing the entanglement entropy of the boundary theory [15-17]. The authors [16,18] defined a candidate $c$-function which extracted from the holographic entanglement entropy of a strip-shape region. In [18], it was shown that the $c$-functions for a Lorentz invariant QFT decreases monotonically if the bulk gravity/matter fields satisfy the null-energy conditions.

There is a natural question that in what extend one can recover monotonic RG flows for theories which exhibit broken Lorentz invariance. In [19], it was shown that for weakly coupled Lorentz-violating field theories, the entanglement entropy does not decrease monotonically under RG flows. On the other hand, [20] claimed that for Lorentzviolating strongly coupled field theories with holographic dual such a breakdown of the candidate $c$ function can be seen. However they also, with respect to the asymptotic UV behavior of the geometry, identified sufficient conditions to have a monotonically decreasing $c$ function along the RG flow. These theories are associated to geometries with a dynamical exponent $z$ and a hyperscaling violation parameter $\theta$.

These dual models are important in studying QFTs with violation of Lorentz invariance and broken rotational symmetry [21-31]. It is worth mentioning that there are studies on RG-flows in theories without Lorentz symmetry while they have Galilean invariance only. There is a possibility to introduce monotonically decreasing quantities in these models which start from a UV fixed point which is not relativistically conformal invariant, so they are anisotropic models with Lifshitz symmetry [32-35].

In this work, we raise the question that how the anisotropy affects the monotonicity of the RG flows, and in what conditions we can define the candidate $c$-function from holographic entanglement entropy for theories that exhibit Lorentz-violating as well as rotational symmetry breaking. We will construct a $c$-function that interpolates between a UV CFT and an IR anisotropic theory.

For this regarding, we consider the cases in which the Lorentz and rotational symmetries are broken only in the IR, while the UV boundary is still conformally invariant, and then examine flowing from a fixed point and, in particular, a $c$-function of the RG flow. There are other related works in this context [36,37].

This paper is organized as follows. In Sec. II, first we briefly review the holographic entanglement entropy of a strip-shaped region in an $A d S$ background, then we derive the Ryu-Takayanagi minimal surface for an anisotropic dilaton-axion model and also in a hyperscaling violation background. In Sec. III, we derive null energy conditions, and propose the candidate $c$-function, for geometries dual to anisotropic QFTs. In Sec. IV, we illustrate our results in some anisotropic backgrounds. Finally we conclude in Sec. V. In Appendix A, we provide more details on calculating the holographic entanglement entropy. To do so, we take the width of the strip in two directions: first, along the isotropic scaling dimensions, then in the anisotropic direction. More details on deriving the RG-flow are provided in Appendix B.

\section{HOLOGRAPHIC ENTANGLEMENT ENTROPY OF ANISOTROPIC MODELS}

In the following subsections, we calculate the holographic entanglement entropy of a strip-shaped region for some anisotropic models. To understand its relationship to the $\mathrm{c}$ function, let us first recall [18] approach, which leads to the identification of the candidate $c$-function by the holographic entanglement entropy of a strip-shaped region for a CFT.

In the UV fixed point we have a CFT, which is dual to $\mathrm{AdS}_{d+2}$ background. Therefore, we can apply RyuTakayanagi (RT) prescription $[15,16]$ to derive the holographic entanglement entropy. For a subregion $V$ on the $d+1$-dimensional boundary field theory, it is given by

$$
S_{\mathrm{EE}}=\frac{\operatorname{Area}(m)}{4 G_{N}},
$$

where $m$ is the minimal surface in $d+2$-dimensional bulk and is homologous to $V$ and $\partial_{m}$ matches the entangling surface $\partial_{V}$ on the boundary. The above formula holds in the case that the bulk physics is described by the Einstein gravity.

The holographic entanglement entropy for a strip-shaped region, where entangling surface is described by two parallel $(d-1)$-dimensional planes separated by a distance $\ell$, is given by $[15,16]$

$$
S_{\mathrm{CFT}}=\alpha_{d}\left(\frac{H}{\delta}\right)^{d-1}-\frac{1}{(d-1) \beta_{d}} C_{d}\left(\frac{H}{\ell}\right)^{d-1}
$$

where $\alpha_{d}$ and $\beta_{d}$ are positive dimensionless numerical factors, and $H \gg \ell$ can be intended as an IR regulator along the entangling surface. The first term denotes the area law and the second one is finite with coefficient $C_{d}$, which is related to the central charge of the underlying CFT via

$$
C_{d}=\beta_{d} \frac{\ell^{d}}{H^{d-1}} \frac{\partial S_{\mathrm{EE}}^{\mathrm{CFT}}}{\partial \ell}
$$

This is suggestive that a $c$-function candidate along the RG flows can be extracted from the holographic entanglement entropy as $[16,18]$

$$
c_{d}=\beta_{d} \frac{\ell^{d}}{H^{d-1}} \frac{\partial S_{\mathrm{EE}}}{\partial \ell} .
$$


where at the fixed point we have $c_{d}=C_{d}$. The monotonicity of this function along the RG flows comes from the Lorentz symmetry and subadditivity inequalities of the entanglement entropy.

In the next section, we promote it to an anisotropic theory and consider the sufficient conditions for monotonically decreasing of the candidate $c$-function along RG flows. Before that, it would be helpful to find the entanglement entropy of a strip-shaped region for such theories. We consider two kinds of anisotropic models.

\section{A. An anisotropic model in the UV regime}

In this subsection, we consider an anisotropic theory for which the dual gravitational theory is defined by the Einstein-axion-dilaton action [21,28,29],

$$
\begin{aligned}
S & =\frac{1}{2 \ell_{p}^{3}} \int d^{5} x \sqrt{-g}\left(R+\mathcal{L}_{M}\right) \\
\mathcal{L}_{M} & =-\frac{1}{2}(\partial \phi)^{2}+V(\phi)-\frac{1}{2} Z(\phi)(\partial \chi)^{2}
\end{aligned}
$$

where $V$ and $Z$ are a potential for the dilaton field $\phi$, and a coupling between the axion field $\chi$ and the dilaton, respectively. With respect to generic choice of the $V$ and $Z$ we can find various solutions. With

$$
V(\phi)=12 \cosh (\sigma \phi)-6 \sigma^{2} \phi^{2}, \quad Z(\phi)=e^{2 \gamma \phi}
$$

near the boundary, this potential approaches the cosmological constant (with the radius of curvature is $L=1$ ) and we have a UV fixed point with conformal symmetry on the boundary.

Now take a linear axion as $\chi=a y$, then the Einstein's equations can be found as

$$
\begin{aligned}
3 A^{\prime}\left(2 A^{\prime}+h^{\prime}\right) & =\frac{1}{4}\left(2 e^{2 A} V(\phi)-a^{2} e^{-2 h} Z(\phi)+\phi^{\prime 2}\right) \\
3 A^{\prime \prime}+3 A^{\prime} h^{\prime}+3 A^{\prime 2}+h^{\prime \prime}+h^{\prime 2} & \\
= & \frac{1}{4}\left(2 e^{2 A} V(\phi)-a^{2} e^{-2 h} Z(\phi)-\phi^{\prime 2}\right)
\end{aligned}
$$

$3 e^{2 h}\left(A^{\prime \prime}+A^{\prime 2}\right)=\frac{1}{4}\left(2 e^{2(A+h)} V(\phi)-e^{2 h} \phi^{\prime 2}+a^{2} Z(\phi)\right)$

and the dilaton equation,

$\phi^{\prime \prime}+\phi^{\prime}\left(3 A^{\prime}+h^{\prime}\right)=a^{2} \gamma e^{2 \gamma \phi-2 h}+12 \sigma e^{2 A}(\sigma \phi-\sinh (\sigma \phi))$.

where an anisotropic ansatz is introduced as

$$
\begin{aligned}
d s^{2} & =e^{2 A(r)}\left[-f(r) d t^{2}+\frac{d r^{2}}{f(r)}+d x_{1}^{2}+d x_{2}^{2}+e^{2 h(r)} d y^{2}\right] \\
\phi & =\phi(r), \quad \chi=a y .
\end{aligned}
$$

Here, we restrict ourselves to zero temperature solution for which $f(r)=1$. For a small anisotropy ar $\ll 1$, a perturbative solution can be found as

$$
\begin{aligned}
A(r)= & -\log (r)-\frac{a^{2} r^{2}}{72}+\frac{a^{4} r^{4}}{1200}\left(3 \gamma^{2}+1\right)(1-5 \log (a r)) \\
& +\mathcal{O}(a r)^{6}, \\
h(r)= & \frac{a^{2} r^{2}}{8}-\frac{a^{4} r^{4}}{2592}\left(31+81 \gamma^{2}-54\left(3 \gamma^{2}+1\right) \log (a r)\right) \\
& +\mathcal{O}(a r)^{6}, \\
\phi(r)= & -\frac{\gamma a^{2} r^{2}}{4}+\frac{a^{4} r^{4}}{96} \gamma\left(3 \gamma^{2}+1\right)(1-4 \log (a r))+\mathcal{O}(a r)^{6} .
\end{aligned}
$$

In the following, we are going to find the entanglement entropy for two cases with the width of strip along one of $x$ coordinates and along $y$ direction.

Let us first consider the width of the strip along one of $x_{i}$ 's directions, say $x=x^{1}$ as $-\ell / 2 \leq x \leq \ell / 2$, then parametrize the RT surface as $r=r(x)$. The entanglement entropy follows as

$$
S_{x}=\frac{4 \pi H^{d-1}}{\ell_{p}^{d}} \int_{0}^{\frac{\ell-\epsilon}{2}} d x e^{B} \sqrt{\dot{r}^{2}+1}
$$

where $B(r)=d A(r)+h(r)$ with $d=3, \epsilon$ is a UV cutoff and $H$ is a cutoff on length of the strip. The EoM is

$$
\ddot{r}=B^{\prime}\left(1+\dot{r}^{2}\right)
$$

where dot denotes $d / d x$ and prime is $d / d r$. Since the integrand of (2.15), $\mathcal{L}$ is independent of $x$, we can introduce the following constant of motion,

$$
\begin{aligned}
K_{d}(\ell) & =\left(\mathcal{L}-\dot{r} \frac{\partial \mathcal{L}}{\partial \dot{r}}\right)^{-1} \\
& =e^{-B} \sqrt{\dot{r}^{2}+1}
\end{aligned}
$$

Then we can write

$$
K_{d}\left(r_{m}\right)=e^{-B\left(r_{m}\right)}
$$

where $r_{m}$ is the maximum of $r$ at $\dot{r}=0$. It follows then 


$$
\begin{aligned}
S_{x} & =\frac{4 \pi H^{d-1}}{\ell_{p}^{d}} \int_{0}^{\frac{\ell-\epsilon}{2}} d x e^{2 B(r(x))-B\left(r_{m}\right)} \\
& =\frac{4 \pi H^{d-1}}{\ell_{p}^{d}} \int_{\delta}^{r_{m}} d r \frac{e^{2 B(r(x))-B\left(r_{m}\right)}}{\sqrt{e^{2\left(B(r)-B\left(r_{m}\right)\right)}-1}}
\end{aligned}
$$

where we used $\dot{r}=-\sqrt{e^{2 B(r)-2 B\left(r_{m}\right)}-1}$ and $\delta$ is the UV cutoff on $r$ coordinate.

We compute the entanglement entropy in the Appendix $\mathrm{A}$ and find it as,

$$
\begin{aligned}
S_{x}= & \frac{4 \pi H^{2}}{\ell_{p}^{3}}\left[-\frac{0.1603}{\ell^{2}}+\frac{a^{2}}{12} \log \ell+0.0316 a^{2}\right. \\
& -\left(0.0088+0.0293 \gamma^{2}+0.0048 \gamma^{4}\right) a^{4} \ell^{2} \\
& \left.+\left(0.0079+0.0127 \gamma^{2}-0.0326 \gamma^{4}\right) a^{4} \ell^{2} \log (a \ell)\right] .
\end{aligned}
$$

The leading term corresponds to (2.2) with $d=3$, but in contrast, the second term in (2.20) is a universal logarithmic term which has no counterpart in (2.2).

As the second case, we consider the strip as $-\ell / 2 \leq$ $y \leq \ell / 2$, and reparametrize the RT surface as $r=r(y)$. Then we have

$$
S_{y}=\frac{4 \pi H^{d-1}}{\ell_{p}^{d}} \int_{0}^{\frac{\ell-\epsilon}{2}} d x e^{d A} \sqrt{\dot{r}^{2}+e^{2 h}} .
$$

The constant of motion is

$$
K_{d}(\ell)=e^{-B} \sqrt{e^{-2 h} \dot{r}^{2}+1}, \quad \Longrightarrow \quad K_{d}\left(r_{m}\right)=e^{-B\left(r_{m}\right)}
$$

It follows then

$$
\begin{aligned}
S_{y} & =\frac{4 \pi H^{d-1}}{\ell_{p}^{d}} \int_{0}^{\frac{\ell-\epsilon}{2}} d y e^{2 B(r(y))-B\left(r_{m}\right)} \\
& =\frac{4 \pi H^{d-1}}{\ell_{p}^{d}} \int_{\delta}^{r_{m}} d r \frac{e^{2 B(r)-B\left(r_{m}\right)}}{e^{-h} \sqrt{e^{2 B(r)-2 B\left(r_{m}\right)}-1}}
\end{aligned}
$$

where we used $\dot{r}=-e^{-h} \sqrt{e^{2 B(r)-2 B\left(r_{m}\right)}-1}$. From the calculations in the Appendix A, we found,

$$
\begin{aligned}
S_{y}= & \frac{4 \pi H^{2}}{\ell_{p}^{3}}\left[-\frac{0.1603}{\ell^{2}}-\frac{a^{2}}{24} \log (\ell)+0.0259 a^{2}\right. \\
& +\left(0.0002+0.0050 \gamma^{2}\right) a^{4} \ell^{2} \\
& \left.-\left(0.0039+0.0118 \gamma^{2}\right) a^{4} \ell^{2} \log (a \ell)\right]
\end{aligned}
$$

\section{B. Anisotropy in a hyperscaling model}

As another interesting case, consider the potential $V$ in (2.5) to be $[21,22]$

$$
V(\phi)=6 e^{\sigma \phi}, \quad Z(\phi)=e^{2 \gamma \phi}
$$

the resulting solution of equation of motions is a Lifshitzlike anisotropic hyperscaling violation metric with the arbitrary critical exponent $z$. The hyperscaling violation exponent $\theta$ is related to the $\sigma$ and $\gamma$ constants. It is worth mentioning that this potential can be considered as the IR limit of (2.6). But, these solutions indeed exist as exact metrics along UV to IR (not only as limits). The Lagrangian setup for these solutions from UV to IR as well as the exact scaling factors of the background solution are presented in $[22,23] .{ }^{1}$ There are other related works in this background and context [27,30]. In otherwise, the anisotropic IR metrics that is derived in [21] can be understood as a double wick rotation of the standard Lifshitz/HsV metrics as well as scaling of $z$ and $\theta$ [31].

In any way, here we suppose it to be an independent potential valid everywhere (not only IR). Now by a linear axion in the $y$-direction, $\chi=a y$, one finds the following exact solution $[21,22]$

$$
\begin{aligned}
d s^{2} & =\tilde{L}^{2}(a r)^{\frac{2 \theta}{d z}}\left(\frac{-d t^{2}+d r^{2}+d \vec{x}_{d-1}^{2}}{(a r)^{2}}+\frac{c_{1} d y^{2}}{(a r)^{2 / z}}\right), \\
\phi & =c_{2} \log (a r)+\phi_{0} .
\end{aligned}
$$

where $z=\left(4 \gamma^{2}-3 \sigma^{2}+2\right) /\left(4 \gamma^{2}-6 \gamma \sigma\right)$ and $\theta=3 \sigma /(2 \gamma)$. For simplicity we consider $a=1$ and absorb $c_{1}$ in $y$ coordinate,

$$
d s^{2}=\tilde{L}^{2} r^{\frac{2 \theta}{d z}}\left(\frac{-d t^{2}+d r^{2}+d \vec{x}_{d-1}^{2}}{r^{2}}+\frac{d y^{2}}{r^{2 / z}}\right) .
$$

The scale transformation of the coordinates is defined as $t \rightarrow \lambda t, \quad r \rightarrow \lambda r \quad x_{i} \rightarrow \lambda x_{i}, \quad y \rightarrow \lambda^{\frac{1}{z}} y, \quad d s^{2} \rightarrow \lambda^{\frac{2 \theta}{d z}} d s^{2}$.

The metric transforms covariantly under these transformations.

The holographic entanglement entropy of a curved region in this background is considered in [30]. The above metric has an anisotropic scaling in one of spatial coordinates, so we consider two possible ways to addressing this problem. First, we take the width of the strip along the anisotropic scaling direction $y$, while in the second case we put up the width of strip along one of the isotropic scaling directions $x_{i}$ 's.

\footnotetext{
${ }^{1}$ We would like to thank Dimitrios Giataganas for pointing this out to us.
} 


\section{The width of the strip along the anisotropic scaling direction}

In this case, we take the strip as

$$
\frac{-\ell}{2} \leq y \leq \frac{\ell}{2}, \quad 0 \leq x_{i} \leq H,
$$

We choose the profile of the bulk minimal surface as $y=y(r)$. Hence, the induced metric on this minimal surface reads

$$
d s^{2}=\tilde{L}^{2} r^{\frac{2 \theta}{d z}-2}\left(d \vec{x}_{d-1}^{2}+\left(1+\frac{y^{2}}{r^{\frac{2}{z}-2}}\right) d r^{2}\right)
$$

where $\tilde{L}$ is the $A d S$ curvature scale, and $y^{\prime}=\partial_{r} y$. The holographic entanglement entropy is given by

$$
\begin{aligned}
S_{\mathrm{EE}}= & \frac{2 \pi}{\ell_{p}^{d}} \int d \sigma \sqrt{\gamma}=\frac{\tilde{L}^{d} 2 \pi H^{d-1}}{\ell_{p}^{d}} \\
& \times \int_{\delta}^{r_{m}} d r \frac{1}{r^{d+\frac{1-\theta}{z}-1}} \sqrt{r^{2\left(\frac{1}{z}-1\right)}+y^{\prime 2}},
\end{aligned}
$$

in which $\delta$ is a $U V$ cutoff. $r_{m}$ is the turning point of the minimal surface defined in such a way that $r_{m}=r(x=0)$ and $y^{\prime}\left(r_{m}\right)=\infty$. Note that the entropy functional (2.31) does not depend explicitly on $y$, so there is a conserved quantity

$$
\frac{1}{r^{d+\frac{1-\theta}{z}-1}} \frac{y^{\prime}}{\sqrt{r^{\frac{2}{2}-2}+y^{\prime 2}}}=\frac{1}{r_{m}^{d+\frac{1-\theta}{z}-1}}
$$

Using this, we can rewrite $S_{\mathrm{EE}}$ and width of the strip $\ell$ as functions of turning point $r_{m}$,

$$
S_{\mathrm{EE}}=\frac{\tilde{L}^{d} 2 \pi H^{d-1}}{\ell_{p}^{d}} I
$$

in which $I$ is

$$
\begin{gathered}
I=\frac{1}{r_{m}^{d-\frac{\theta}{2}-1}} \int_{\frac{\delta}{r_{m}}}^{1} d u \frac{u^{\frac{\theta}{z}-d}}{\sqrt{1-u^{2\left(d+\frac{1-\theta}{z}-1\right)}}}, \\
\ell=2 r_{m}^{\frac{1}{2}} \int_{0}^{1} d u \frac{u^{d+\frac{2-\theta}{z}-2}}{\sqrt{1-u^{2\left(d+\frac{1-\theta}{2}-1\right)}}},
\end{gathered}
$$

where $u=r / r_{m}$. If we explicitly compute the above integrals, we can write the $S_{\mathrm{EE}}$ as a function of width of the strip $\ell$. To proceed, we must note that for special value of $\theta$ the logarithmic term appears. So, we separate it. (i) $\theta \neq z(d-1)$

$$
\begin{gathered}
\ell=\alpha r_{m}^{\frac{1}{2}}, \quad \alpha=2 \sqrt{\pi} z \frac{\Gamma\left(\frac{z(d-1)+2-\theta}{2(z(d-1)+1-\theta)}\right)}{\Gamma\left(\frac{1}{(2(z(d-1)+1-\theta))}\right)} \\
S_{\mathrm{EE}}=\frac{4 \pi z H^{\frac{\theta}{z}}}{z(d-1)-\theta} \frac{\tilde{L}^{d}}{\ell_{p}^{d}}\left(\frac{H}{\delta}\right)^{d-\frac{\theta}{z}-1} \\
-\beta_{d} \frac{\tilde{L}^{d}}{\ell_{p}^{d}}\left(\frac{H}{\ell}\right)^{z(d-1)-\theta}
\end{gathered}
$$

where

$$
\begin{aligned}
\beta_{d}= & \frac{2 z \pi^{\frac{3}{2}}}{2 z(d-1)-2 \theta+1} H^{(1-z)(d-1)+\theta} \\
& \times\left(\frac{\Gamma\left(-\frac{1}{2}+\frac{1}{2(z(d-1)+1-\theta)}\right)}{\Gamma\left(-1+\frac{1}{(2(z(d-1)+1-\theta))}\right)}\right) \alpha^{(d-1) z-\theta} .
\end{aligned}
$$

(ii) $\theta=z(d-1)$

In this case, we find $\ell=2 z r_{m}^{\frac{1}{2}}$, and the holographic entanglement entropy is

$$
S_{\mathrm{EE}}=2 \pi H^{d-1} \frac{\tilde{L}^{d}}{\ell_{p}^{d}} \log \frac{\ell^{z}}{(2 z)^{z} \delta} .
$$

This result shows that the boundary theory exhibits a logarithmic area law violation, which may be a sign that the boundary theory has a Fermi surface.

\section{The width of the strip along one of the isotropic scaling direction}

In this case, we take the strip as

$$
\frac{-\ell}{2} \leq x_{1} \leq \frac{\ell}{2}, \quad 0 \leq x_{i} \leq H, \quad i \neq 1, \quad 0 \leq y \leq H,
$$

We choose the profile of the bulk minimal surface as $x_{1}=x(r)$. So the induced metric on the minimal surface is derived as

$d s^{2}=\tilde{L}^{d{ }^{d z}-2}\left(d \vec{x}_{d-2}^{2}+\frac{1}{r^{2}-2} d y^{2}+\left(1+x^{\prime 2}\right) d r^{2}\right)$,

where $\tilde{L}$ is the $A d S$ curvature scale, and $x^{\prime}=\partial_{r} x$. The holographic entanglement entropy is given by

$S_{\mathrm{EE}}=\frac{2 \pi}{\ell_{p}^{d}} \int d \sigma \sqrt{\gamma}=\frac{\tilde{L}^{d} 2 \pi H^{d-1}}{\ell_{p}^{d}} \int_{\delta}^{r_{m}} d r \frac{\sqrt{1+x^{\prime 2}}}{r^{d+\frac{1-\theta}{z}-1}}$,

in which $\delta$ is a $U V$ cutoff, and $r_{m}$ is turning point of minimal surface, and $x^{\prime}\left(r_{m}\right)=\infty$. The corresponding conserved quantity is defined as 


$$
\frac{1}{r^{d+\frac{1-\theta}{z}-1}} \frac{x^{\prime}}{\sqrt{1+x^{\prime 2}}}=\frac{1}{r_{m}^{d+\frac{1-\theta}{z}-1}}
$$

Using this we can rewrite the $S_{\mathrm{EE}}$ and width of the strip $\ell$ as functions of turning point $r_{m}$,

$$
S_{\mathrm{EE}}=\frac{\tilde{L}^{d} 2 \pi H^{d-1}}{\ell_{p}^{d}} I
$$

in which $I$ is

$$
\begin{gathered}
I=\frac{1}{r_{m}^{d+\frac{1-\theta}{z}-2}} \int_{\frac{\delta}{r_{m}}}^{1} d u \frac{1}{u^{d+\frac{1-\theta}{z}}-1} \frac{1}{\sqrt{1-u^{2\left(d+\frac{1-\theta}{z}-1\right)}}}, \\
\ell=2 r_{m} \int_{0}^{1} d u \frac{u^{d+\frac{1-\theta}{z}-1}}{\sqrt{1-u^{2\left(d+\frac{1-\theta}{z}-1\right)}}},
\end{gathered}
$$

Here $u=r / r_{m}$. Similarly, we can explicitly compute the above integrals, and rewrite the $S_{\mathrm{EE}}$ as a function of width of the strip $\ell$. Again, for a special value of $\theta$ a logarithmic term appears. So, we separate it.

(i) $\theta \neq z(d-2)+1$

$$
\begin{gathered}
\ell=\alpha r_{m}, \quad \alpha=2 \sqrt{\pi} \frac{\Gamma\left(\frac{z d+1-\theta}{2(z(d-1)+1-\theta)}\right)}{\Gamma\left(\frac{z}{(2(z(d-1)+1-\theta))}\right)} \\
S_{\mathrm{EE}}=\frac{4 \pi z}{z(d-2)+1-\theta} \frac{\tilde{L}^{d}}{\ell_{p}^{d}}\left(\frac{H}{\delta}\right)^{d+\frac{1-\theta}{z}-2} \\
-\beta_{d} \frac{\tilde{L}^{d}}{\ell_{p}^{d}}\left(\frac{H}{\ell}\right)^{d+\frac{1-\theta}{z}-2}
\end{gathered}
$$

where

$$
\begin{aligned}
\beta_{d}= & \frac{2 z \pi^{\frac{3}{2}}}{z(d-2)+1-\theta} H^{1-\frac{1}{z}} \\
& \times\left(\frac{\Gamma\left(\frac{(2-d) z+\theta-1}{2(z(d-1)+1-\theta)}\right)}{\Gamma\left(\frac{z}{(2(z(d-1)+1-\theta))}\right)}\right) \alpha^{\frac{(d-2) z+1-\theta}{z}} .
\end{aligned}
$$

(ii) $\theta=z(d-2)+1$

In this case, we find $\ell=2 r_{m}$, and the holographic entanglement entropy takes

$$
S_{\mathrm{EE}} \sim \frac{\tilde{L}^{d}}{\ell_{p}^{d}} \log \frac{\ell}{\delta}
$$

This result shows that the entanglement entropy of the boundary theory exhibits a logarithmic violation area law, which may be a sign that the boundary theory has a Fermi surface.

\section{A $c$-FUNCTION FROM THE HOLOGRAPHIC ENTANGLEMENT ENTROPY IN AN ANISOTROPIC FIELD THEORY}

In this section, we probe the monotonicity of a candidate $c$ function. Let us start with the following generic metric which exhibits violation of the Lorentz invariance and broken rotational symmetry, but respects translational symmetry,

$$
d s^{2}=e^{2 A(r)}\left(-d t^{2}+d r^{2}+d x_{d-1}^{2}+e^{2 h(r)} d y^{2}\right)
$$

In Sec. IV, we give an explicit example for such theories. To introduce the $c$-function, we consider a strip as the entangled region. Similar to the previous section, we study two cases where the strip is along one of $x_{i}$ 's and along $y$ direction in the following two subsections.

\section{A. The width of strip along one of the isotropic scaling dimensions}

Now we need to derive the variation of $S$ with respect to $\ell$. We follow [18] and details are left to the Appendix B. It results to

$$
\frac{d S_{x}}{d \ell}=\frac{2 \pi H^{d-1}}{\ell_{p}^{d}} \frac{1}{K_{d}}
$$

Then define

$$
\begin{aligned}
c_{x} & :=\beta_{x} \frac{\ell^{d}}{H^{d-1}} \frac{\partial S_{x}}{\partial \ell} \\
& =\beta_{x} \frac{2 \pi \ell^{d}}{\ell_{p}^{d} K_{d}}
\end{aligned}
$$

where $\beta_{x}$ is a positive numerical constant. We then find the flow $d c_{x} / d r_{m}$ where $r_{m}$ is defined as $r_{m}=r(0, \ell)$ with $\dot{r}(0, \ell)=0$ as

$$
\frac{d c_{x}}{d r_{m}}=\beta_{x} \frac{4 \pi \ell^{d-1}}{\ell_{p}^{d}} B^{\prime}\left(r_{m}\right) \int_{0}^{\ell / 2} d x \frac{B^{\prime 2}-d B^{\prime \prime}}{B^{\prime 2}}
$$

The details are given in the Appendix B. Note that by setting $h(r)=0$ and changing the coordinate $r$ to domain wall coordinate, (3.4) transforms to the similar expression given in [18].

To study the behavior of (3.4) under the RG-flow, we use the null energy conditions (NECs). First, let us take a general null vector as

$$
\xi=e^{-A}\left[a_{0} \partial_{t}+a_{r} \partial_{r}+\sum_{i}^{d-1} a_{i} \partial_{i}+e^{-h} a_{y} \partial_{y}\right]
$$

with $a_{0}^{2}=a_{r}^{2}+a_{y}^{2}+\sum_{i=1}^{d-1} a_{i}^{2}$. Then, the general NEC can be read as 


$$
\begin{aligned}
& d a_{r}^{2} A^{\prime 2}-d a_{y}^{2} A^{\prime} h^{\prime}-\left(a_{r}^{2}+a_{y}^{2}\right) h^{\prime 2}-d a_{r}^{2} A^{\prime \prime} \\
& -\left(a_{r}^{2}+a_{y}^{2}\right) h^{\prime \prime} \geq 0
\end{aligned}
$$

One can put $a_{r}=1$ and $a_{y}=0$ to obtain

$$
N_{1} \equiv d A^{\prime 2}-h^{\prime 2}-d A^{\prime \prime}-h^{\prime \prime} \geq 0
$$

Another independent null energy condition can be found by taking $a_{r}=0$ and $a_{y}=1$,

$$
N_{2} \equiv-d A^{\prime} h^{\prime}-h^{\prime 2}-h^{\prime \prime} \geq 0
$$

The inequalities (3.7) and (3.8) can be respectively transformed to

$$
\begin{gathered}
f_{1}^{\prime}(r) \equiv\left(-B^{\prime} e^{-d A+h}\right)^{\prime}=\left(d(d-1) A^{\prime 2}+N_{1}\right) e^{-d A+h} \geq 0 \\
f_{2}^{\prime}(r) \equiv\left(-h^{\prime} e^{B}\right)^{\prime} \geq 0 .
\end{gathered}
$$

Now let us write the integrand of (3.4) as

$$
B^{\prime 2}-d B^{\prime \prime}=d N_{1}+(d-1) h^{\prime 2}+2 h^{\prime} B^{\prime} .
$$

Since the first two terms in the right-hand side are positive, it is enough to put $h^{\prime} B^{\prime} \geq 0$ to have sufficient condition for the integrand to be non-negative. Now (3.9) and (3.10) indicate that if one starts with positive $f_{1}(r)$ and $f_{2}(r)$ at the UV boundary, they remain positive in the bulk which means that we have $B^{\prime}(r) \leq 0$ and $h^{\prime}(r) \leq 0$ everywhere. Then from (3.11) the integrand of (3.4) is positive while $B^{\prime}\left(r_{m}\right) \leq 0$ which in turn implies that the $c$-function is a monotonically decreasing function of $r_{m}$,

$$
\frac{d c_{d}}{d r_{m}} \leq 0
$$

So using the NECs, the sufficient conditions are $B^{\prime}(r) \leq 0$ and $h^{\prime}(r) \leq 0$ at the UV boundary.

To satisfy (3.12), another possibility is demanding $B^{\prime} \geq 0$ and $B^{\prime 2}-d B^{\prime \prime} \leq 0$. To have positive $B^{\prime}$, it is sufficient to have $f_{1} \leq 0$ at the IR limit. We also assume $B^{\prime \prime} \geq 0$ which implies $B^{\prime}$ remains positive. Then

$B^{\prime 2}-d B^{\prime \prime}=-N_{1}-(d+1) B^{\prime \prime}+2 B^{\prime 2}-2 B^{\prime} h^{\prime}-(d-1) h^{\prime 2}$

Since $N_{1}$ and $B^{\prime \prime}$ are positive, the sufficient condition is

$$
2 B^{\prime 2}-2 B^{\prime} h^{\prime}-(d-1) h^{\prime 2} \leq 0
$$

Reminding our assumption $B^{\prime} \geq 0$, the above inequality implies either $h^{\prime} \geq B^{\prime}(\sqrt{(2 d-1)}-1) /(d-1)$ or $h^{\prime} \leq$ $B^{\prime}(-\sqrt{(2 d-1)}-1) /(d-1)$. The corresponding region in the $B^{\prime}-h^{\prime}$ plane is shown in Fig. 1. Unfortunately, neither IR nor UV boundary conditions are sufficient to satisfy these inequalities.

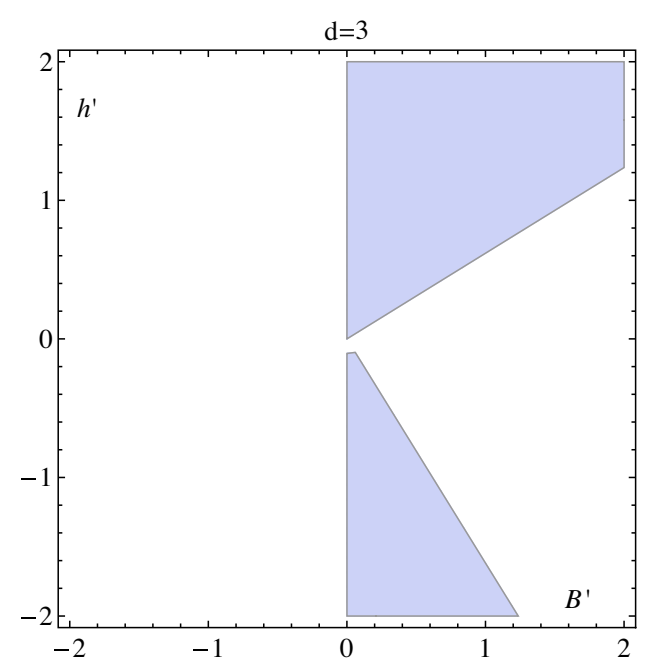

FIG. 1. The shaded area in $B^{\prime}-h^{\prime}$ plane in $d=3$ show the regions where both $B^{\prime} \geq 0$ and (3.8) are satisfied. We also assumed $B^{\prime \prime} \geq 0$.

\section{B. The width of strip along the anisotropic direction}

In this subsection, we consider the width of strip to be along $y$-direction (i.e., $-\ell / 2 \leq y \leq \ell / 2$ ). It is convenient to change variable to $d \rho=e^{-h} d r$, then the metric read as $d s^{2}=e^{2 A(\rho)+2 h(\rho)}\left(-e^{-2 h(\rho)} d t^{2}+d \rho^{2}+e^{-2 h(\rho)} d x_{d-1}^{2}+d y^{2}\right)$

By taking $\rho=\rho(y)$ as the Ryu-Takayanagi (RT) surface, the entropy functional would be

$$
S_{y}=\frac{4 \pi H^{d-1}}{\ell_{p}^{d}} \int_{0}^{\frac{\ell-\epsilon}{2}} d y e^{d A+h} \sqrt{\dot{\rho}^{2}+1}
$$

where $\dot{\rho}=d \rho / d y$. This is similar to (2.15) by replacing $r \rightarrow \rho$ and everything should go exactly the same as previous subsection to find,

$\frac{d c_{y}}{d \rho_{m}}=\tilde{\beta}_{y} \frac{4 \pi \ell^{d-1}}{\ell_{p}^{d}} B^{\prime}\left(\rho_{m}\right) \int_{0}^{\frac{\ell}{2}} d y \frac{B^{\prime}(\rho)^{2}-d B^{\prime \prime}(\rho)}{B^{\prime}(\rho)^{2}}$

Now we go back to $r$ coordinate,

$\frac{d c_{y}}{d r_{m}}=\tilde{\beta}_{y} \frac{4 \pi \ell^{d-1}}{\ell_{p}^{d}} B^{\prime}\left(r_{m}\right) \int_{0}^{\frac{\ell}{2}} d y \frac{B^{\prime 2}-d\left(h^{\prime} B^{\prime}+B^{\prime \prime}\right)}{B^{\prime 2}}$

where all functions are in $r$ coordinate.

Similar to previous subsection, we first consider $B^{\prime} \leq 0$ while the integrand in (3.18) is positive. Then similar to (3.7), we can write

$B^{\prime 2}-d\left(B^{\prime \prime}+h^{\prime} B^{\prime}\right)=d N_{1}+(d-1) h^{\prime 2}+(2-d) h^{\prime} B^{\prime}$ 


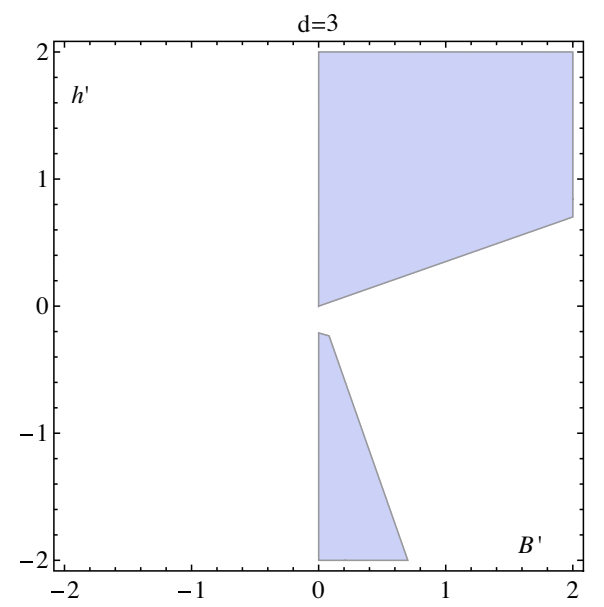

FIG. 2. This diagram is related to the case that the width of strip is along $y$-direction with $d=3$. The shaded area in $B^{\prime}-h^{\prime}$ plane, shows the regions where both $B^{\prime} \geq 0$ and (3.21) are valid. We also assumed $B^{\prime \prime} \geq 0$.

For $d=2$, this is always positive. For $d>2$ and since we assumed $B^{\prime} \leq 0$, the sufficient condition is $h^{\prime} \geq 0$. Equation (3.9) indicates that if starting with a negative $B^{\prime}$ at UV boundary, it remains negative in whole regions inside the bulk. In contrast, there is no guarantee for $h^{\prime}$ to remain positive starting with $h^{\prime}>0$ at $r=0$. However, we show that our example in Sec. IVA satisfies conditions $B^{\prime}<0, \quad h^{\prime}>0$ and gives monotonically decreasing $c$-function in some range of parameters.

Now consider the case, when $B^{\prime}\left(r_{m}\right) \geq 0$ and the integrand of (3.17) is negative. We can write

$$
\begin{aligned}
& B^{\prime 2}-d\left(B^{\prime \prime}+B^{\prime} h^{\prime}\right)=-d N_{1}-2 d B^{\prime \prime}+2 B^{\prime 2} \\
& -B^{\prime}(d+2) h^{\prime}-(d-1) h^{\prime 2}
\end{aligned}
$$

Again we assume $B^{\prime \prime} \geq 0$ and demand the right-hand side of the above equation to be negative. It is sufficient to set

$$
2 B^{\prime 2}-B^{\prime}(d+2) h^{\prime}-(d-1) h^{\prime 2} \leq 0
$$

The corresponding region in the $B^{\prime}-h^{\prime}$ plane is shown in Fig. 2.

\section{SOME EXAMPLES}

To ilustrate our RG-flow in (3.4), we consider the axiondilaton and hyperscaling models discussed in Sec. II.

\section{A. $c$-function for the axion-dilaton model}

Let us consider the small $a$ expansion in (2.12) to find

$$
\begin{aligned}
B(r)= & -3 \log (r)+\frac{a^{2} r^{2}}{12}-\frac{a^{4} r^{4}}{64800}\left(1539 \gamma^{2}+613\right) \\
& +\frac{a^{4} r^{4}}{120}\left(3 \gamma^{2}+1\right) \log (a r)+\cdots
\end{aligned}
$$

It indicates that near UV boundary, $B^{\prime}(r)<0$, while from (2.12) we have $h^{\prime}(r)>0$, so the sufficient conditions discussed below (3.11) are not satisfied. However, we can investigate the behavior of $d c / d r_{m}$ explicitly. For the width of strip along the $x$ direction, the integrand of (3.4) can be found as

$$
\begin{aligned}
\frac{B^{\prime 2}-d B^{\prime \prime}}{B^{\prime 2}}= & -\frac{a^{2} r^{2}}{6}-\frac{a^{4} r^{4}}{972}\left(54\left(3 \gamma^{2}+1\right) \log (\text { ar })\right. \\
& \left.-81 \gamma^{2}-22\right)+\cdots
\end{aligned}
$$

which is negative near UV boundary while $B^{\prime}(r)<0$, therefore we have $d c_{x} / d r_{m}>0$ which has a wrong sign. In Fig. 3 left panel, the shaded area shows the region where $B^{\prime 2}-d B^{\prime \prime}$ and $B^{\prime}$ have opposite signs. It indicates that $d c_{x} / d r_{m}$ doesn't have any monotonic decreasing behavior. From the calculations in Appendix A, we found $S_{\mathrm{EE}}$ and $d c / d \ell$ as a function of $\ell$ and draw them in Fig. 4. It shows that $d c / d \ell$ cannot be negative in the UV regime.

For the width of strip along $y$-direction, the sufficient condition from (3.19) is $B^{\prime} \leq 0$ and $h^{\prime} \geq 0$. These are valid close to the UV boundary. To be sure about the behavior inside the bulk, let us compute the integrand of (3.18) as,

$$
\begin{aligned}
\frac{B^{\prime 2}-d\left(B^{\prime \prime}+h^{\prime} B^{\prime}\right)}{B^{\prime 2}}= & \frac{a^{2} r^{2}}{12}+\frac{a^{4} r^{4}}{3888}\left(37+81 \gamma^{2}\right. \\
& \left.+108\left(3 \gamma^{2}+1\right) \log (a r)\right)+\cdots
\end{aligned}
$$

It is positive close to $r=0$ and since $B^{\prime}<0$ in this region one expects $d c_{y} / d r_{m}$ to be negative. However, the right panel in Fig. 3 shows that this is valid in the region where $N E C$ 's are valid and $\gamma \leq 3$. For $\gamma>3$, there is a narrow region where $N E C$ 's are valid, but the integrand of (3.18) is negative, which leads to the wrong sign $d c_{y} / d r_{m}>0$. One may claim that the integration in (3.18) from 0 to $r_{m}$ may gives an overall positive result, but the explicit investigation shows that this is not the case.

To summarize the results in this subsection, we found that the behavior of $d c_{x} / d r_{m}$ does not decrease monotonically when the strip width is along the $x$-direction. In the case of $y$-direction, $d c_{y} / d r_{m}<0$ as long as NEC's are valid and $\gamma \leq 3$. Explicit calculation of $c$-functions and its derivatives are given in Appendix A and depicted in Fig. 4.

\section{B. $\boldsymbol{c}$-function for the hyperscaling model}

Here, we consider the anisotropic model (2.5) with the potential (2.25), which as discussed in the beginning of Sec. II B, is valid in the full range of scales from UV to IR. However, it can also be considered as the IR limit of (2.6). This later point of view is more convenient to study the $c$-function, since our results in Sec. II B indicate that the entanglement entropy behaves as $S \sim \ell^{1-n}$, where $n \neq d$ 

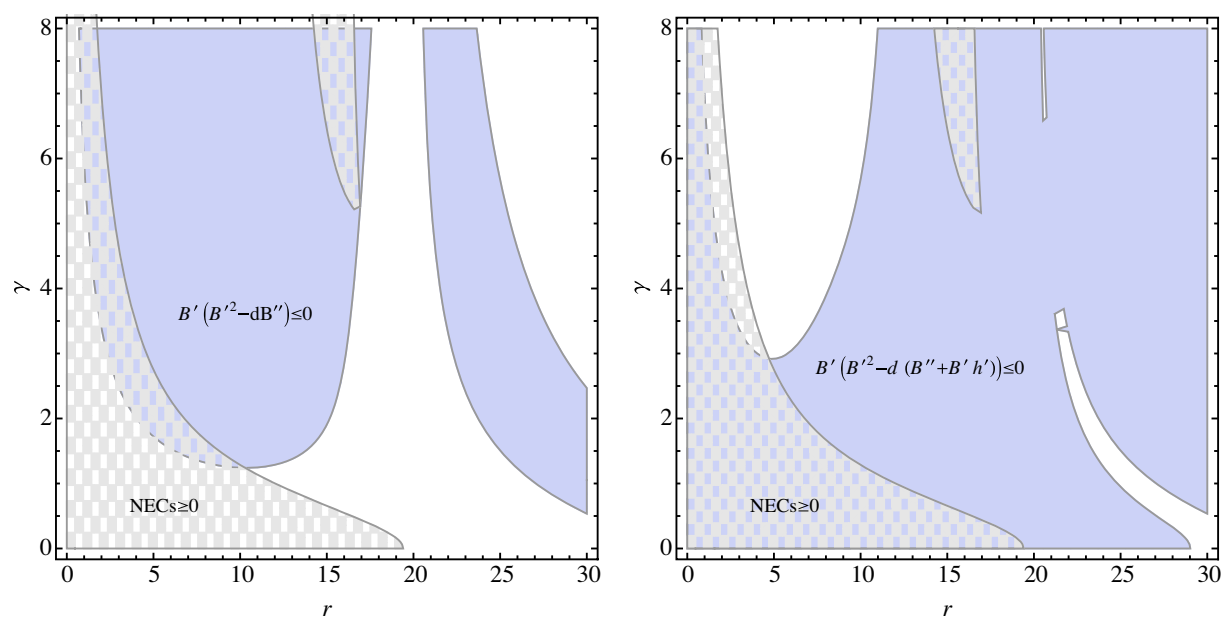

FIG. 3. In the left panel, shaded areas in $r-\gamma$ plane depict the regions where $B^{\prime 2}-d B^{\prime \prime}$ and $B^{\prime}$ have opposite signs. This is sufficient to have $d c_{x} / d r_{m} \leq 0$ for the width of strip along the $x$ direction. The shaded area in the right panel shows $d c_{y} / d r_{m} \leq 0$ for width of strip along the $y$ direction. The checkered regions in both panels show the area where $N E C^{\prime} s$ are valid. It can be inferred that $d c_{x} / d r_{m}$ has no monotonic behavior, whereas, $d c_{y} / d r_{m}$ for $\gamma<3$ is monotonically decreasing as long as $N E C$ 's are valid.

and depends on $d, \theta$ and $z$, so the $c$-function in (2.4) does not approach to the CFT central chrage in the UV limit. As such, we consider (2.25) as the IR tail of the anisotropic theory and investigate the conditions for which the $c$-function (2.4) is monotonically decreasing along the RG-flow in this regime.
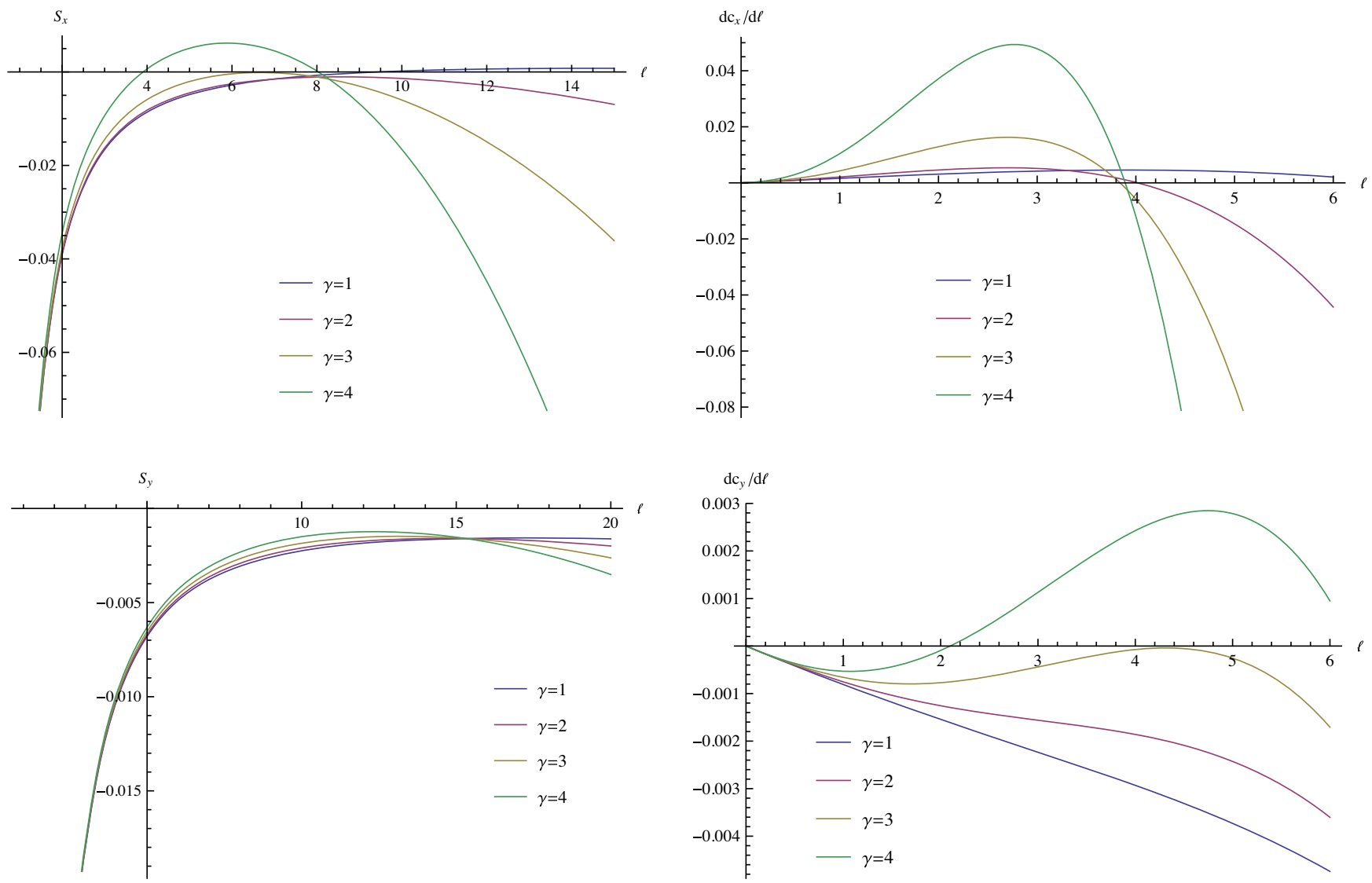

FIG. 4. The above (below) panels are the entropy $S$ and the derivative of the $c$-function for the width of strip in the $x$-direction ( $y$-direction) as derived in Appendix A. All diagrams are drawn for $a=0.1$ and $\gamma=1,2,3,4$. For the $x$ direction, there is no monotonically decreasing of the $c_{x}$-function, while in the $y$ direction we can see the monotonic behavior with negative derivative for $\gamma \leq 3$. 
Let us recall the solution for arbitrary $d$ as [21],

$$
d s^{2}=\tilde{L}^{2} r^{\frac{2 \theta}{d z}}\left(\frac{-d t^{2}+d r^{2}+d \vec{x}_{d-1}^{2}}{r^{2}}+\frac{d y^{2}}{r^{\frac{2}{2}}}\right),
$$

In comparison to (3.1), we identify metric functions as

$$
\begin{aligned}
A(r) & =\left(\frac{\theta}{d z}-1\right) \log (r) \\
h(r) & =\left(1-\frac{1}{z}\right) \log (r)
\end{aligned}
$$

It follows then,

$$
\begin{gathered}
B^{\prime}=\frac{-d z+\theta+z-1}{z} \frac{1}{r} \\
I_{x}:=\frac{B^{\prime 2}-d B^{\prime \prime}}{B^{\prime 2}}=\frac{d z}{-d z+\theta+z-1}+1
\end{gathered}
$$

It is clear that both expressions have definite signs independent of $r$. So we can set parameters such that either $B^{\prime}$ and $I_{x}$ have opposite signs and they never change their signs as functions of $r$. In this regime, the null energy conditions (NEC) are as follows

$$
\begin{aligned}
& -d(\theta-1) z-d+\theta^{2} \geq 0 \\
& (z-1)(d z-\theta+1) \geq 0 .
\end{aligned}
$$

Figure 5 left panel, shows regions in $\theta-z$ plane where $B^{\prime}$ and $I_{x}$ have opposite signs and NEC's are satisfied which guarantees that $c_{x}$ is a monotonically decreasing function of $r_{m}$. For the width of strip along the $y$-direction, we find the integrand of (3.18) as

$I_{y}:=\frac{B^{\prime 2}-d\left(B^{\prime \prime}+h^{\prime} B^{\prime}\right)}{B^{\prime 2}}=\frac{z}{-d z+\theta+z-1}+1$.

Now we look for regions where $B^{\prime}$ and $I_{y}$ have opposite signs which are the shaded regions shown in Fig. 5 right panel. This diagram shows that for $z>0$ and $\theta<0$, the NEC's $\geq 0$ are a subset of the shaded region which indicates that at least for $z>0$ and $\theta<0$, the null energy conditions are sufficient to have a monotonically decreasing $c$-function.

\section{CONCLUSION}

Within gauge/gravity duality, we calculate the entanglement entropy (EE) of a strip-shaped region in $d+1$ dimensional quantum field theory (QFT) with broken Lorentz invariance as well as rotational symmetry. These theories are representative for many physical phenomena. So, extracting various information from these theories may be helpful in understanding of some quantum systems. One of the ways to receive information is computing the entanglement entropy, which is a measure of entanglement structure of the ground state of underlying theory.

We have computed the entanglement entropy of anisotropic strongly gauge fields which are dual to two classes of geometries. The first class is an anisotropic dilaton-axion background and the second one is identified with dynamical and hyperscaling violation parameters $\{z, \theta\}$. Note, these geometries are defined in some intermediate regime.
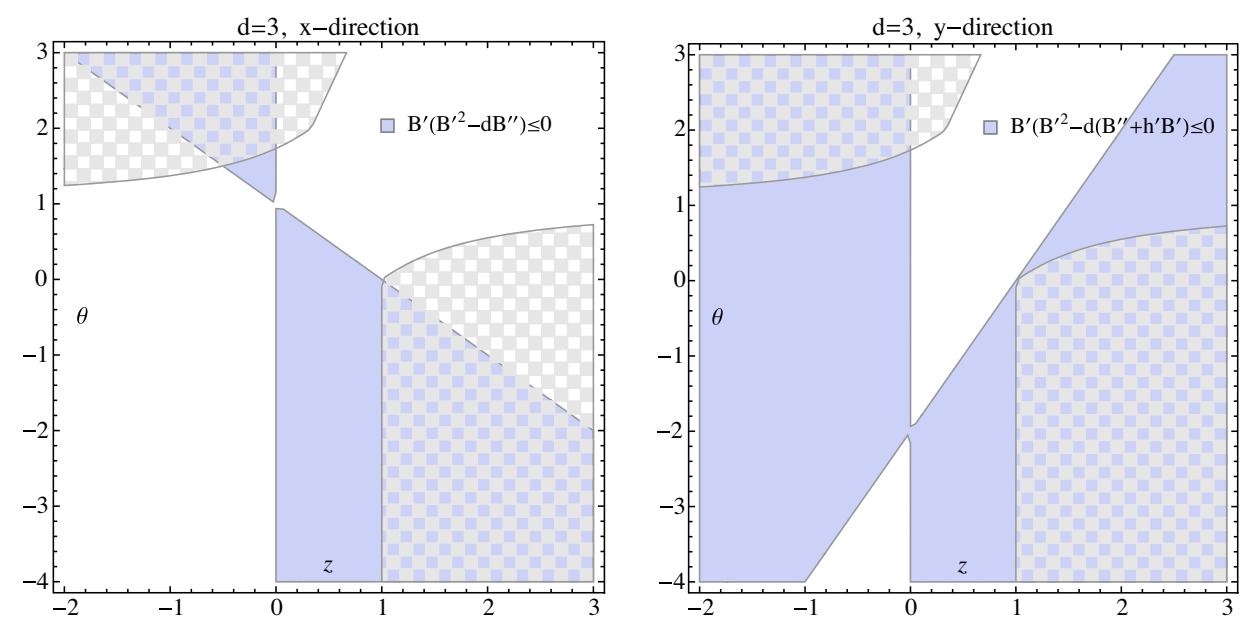

FIG. 5. The left (right) panel is for the width of in the $x$-direction ( $y$-direction) and the shaded areas in $\theta-z$ plane correspond to regions where $B^{\prime}$ and Eq. (4.8) [Eq. (4.11)] have opposite signs hence $d c / d r_{m} \leq 0$. The checkered areas show the regions where $N E C$ 's are valid. We set $d=3$. 
We have done analytical computations in order to derived the holographic entanglement entropy of a strip-shaped region.

The holographic entanglement entropy of strip-shaped region has been used to construct a candidate $c$-function which is interpolated between UV and IR fixed points. We identified sufficient conditions to provide monotonically decreasing of $c$ along RG flows. These conditions are extracted from the null energy conditions of the bulk matter fields.

We considered two cases where the width of strip is along one of the isotropic directions or along the anisotropic one. In these two cases different conditions are needed to provide the monotonicity of the $c$-function. For a strip with width along the anisotropic direction, the null energy conditions provided with suitable UV boundary conditions are sufficient to have a monotonically decreasing $c$-function.

In order to find an intuition of these general discussion, we illustrated the $c$ function in some special cases in five dimension. We have chosen two cases: near UV, and near IR expansions. For each one we observed that $c$-function monotonically decreased if the null energy conditions are satisfied. Orientation of the strip is also important. For the width of strip along the anisotropic direction the NEC's are enough to have a monotonically decreasing $c$-function, while for the other directions it is not so. This behavior is not so surprising, since the monotonicity of $c$-function is expected from the Lorentz symmetry and subadditivity of the entanglement entropy. For an anisotropic theory the Lorentz symmetry is violated and there is no guarantee for monotonicity of the $c$-function.

It is worth notice that for theories that undergo a confinement/deconfinement transition, the behavior of
$S(\ell)$ has generically a swallowtail behavior [27], (although this behavior should be present as well in the models [21] for large values of sigma, where one also expects a deconfinement transition). Some portions of the swallowtail have $d S / d \ell<0, d^{2} S / d \ell^{2}>0$. However, one can use the $c$-function argument to discard these branches. Indeed, one can show that the physical branches have both $c>0$ and $d c / d \ell<0$ even though $d c / d \ell$ is discontinuous at the point of the transition. ${ }^{2}$

It would be also interesting to consider theories for which, in addition to the Lorentz-violation and broken rotation, there is no translation symmetries.

\section{ACKNOWLEDGMENTS}

Special thanks to Juan F. Pedraza, for motivating this problem, useful comments and discussions. Authors also thank him for his generosity in sharing his perturbative calculations in Sec. II A. We would like to thank Dimitrios Giataganas for useful comments and discussions. M. G. would like to thank Sepideh Mohammadi for encouragement and valuable comments.

Note added.-While this article was being completed, we received [38] which has some overlap with our results.

\section{APPENDIX A: COMPUTING THE ENTANGLEMENT ENTROPY}

Starting with the entanglement region along one of the $x$ - coordinates, we insert the solution (2.12) in (2.19) and expand in powers of $a$. Let us change the variable $u=r / r_{m}$ and subtract and add the divergent part as follows

$$
\begin{aligned}
S_{x}= & \frac{4 \pi H^{2}}{\ell_{p}^{3}} r_{m} \int_{0}^{1} d u\left[\frac{e^{2 B\left(r_{m} u\right)-B\left(r_{m}\right)}}{\sqrt{e^{2\left(B\left(r_{m} u\right)-B\left(r_{m}\right)\right)}-1}}-\left(\frac{a^{2}}{12 r_{m} u}+\frac{1}{r_{m}^{3} u^{3}}\right)\right] \\
& +\frac{4 \pi H^{2}}{\ell_{p}^{3}} r_{m} \int_{\delta / r_{m}}^{1} d u\left(\frac{a^{2}}{12 r_{m} u}+\frac{1}{r_{m}^{3} u^{3}}\right) .
\end{aligned}
$$

The first integral can be expanded to

$$
\frac{4 \pi H^{2}}{\ell_{p}^{3}}\left[\frac{1}{r_{m}^{2}} S_{1}+a^{2} S_{2}+a^{4} r_{m}^{2} S_{3}+a^{4} r_{m}^{2} S_{4} \log \left(a r_{m}\right)\right]
$$

where $S_{i}$ 's are defined as follows and can be found by numerical integration,

\footnotetext{
${ }^{2}$ We would like to thank Juan F. Pedraza for pointing this out to us.
} 


$$
\begin{aligned}
S_{1}= & \int_{0}^{1} d u\left[\frac{1}{\sqrt{\frac{1}{u^{6}}-1} u^{6}}-\frac{1}{u^{3}}\right]=0.2844 \\
S_{2}= & \int_{0}^{1} d u\left[\frac{2 u^{4}+u^{2}+1}{12 \sqrt{\frac{1}{u^{6}}-1} u^{4}\left(u^{4}+u^{2}+1\right)}-\frac{1}{12 u}\right]=0.0351 \\
S_{3}= & \int_{0}^{1} d u\left[\frac{\left(1-u^{2}\right)\left(326 u^{8}+1552 u^{6}+1940 u^{4}+939 u^{2}+388\right)}{64800 \sqrt{\frac{1}{u^{6}}-1}\left(u^{2}-1\right)\left(u^{5}+u^{3}+u\right)^{2}}\right. \\
& +\frac{\gamma^{2}\left(1-u^{2}\right)\left(3078 u^{8}+6156 u^{6}+7695 u^{4}+4617 u^{2}+1539\right)}{64800 \sqrt{\frac{1}{u^{6}}-1}\left(u^{2}-1\right)\left(u^{5}+u^{3}+u\right)^{2}} \\
& \left.+\frac{540\left(2 u^{10}+2 u^{8}+2 u^{6}-u^{4}-u^{2}-1\right)\left(1+3 \gamma^{2}\right) \log (u)}{64800 \sqrt{\frac{1}{u^{6}}-1}\left(u^{2}-1\right)\left(u^{5}+u^{3}+u\right)^{2}}\right]=-0.0073-0.0294 \gamma^{2} \\
S_{4}= & \int_{0}^{1} d u\left[\frac{\left(2 u^{5}+2 u^{3}+u\right)\left(1+3 \gamma^{2}\right)}{120\left(u^{4}+u^{2}+1\right) \sqrt{1-u^{6}}}\right]=0.0085\left(1+3 \gamma^{2}\right)
\end{aligned}
$$

and the second integral in (A1) is found to be

$$
\frac{4 \pi H^{2}}{\ell_{p}^{3}} r_{m} \int_{\delta / r_{m}}^{1} d u\left(\frac{a^{2}}{12 r_{m} u}+\frac{1}{r_{m}^{3} u^{3}}\right)=\frac{4 \pi H^{2}}{\ell_{p}^{3}}\left(\frac{1}{2 \delta^{2}}-\frac{1}{2 r_{m}^{2}}-\frac{1}{12} a^{2} \log \left(\frac{\delta}{r_{m}}\right)\right) .
$$

Putting all results together

$$
\begin{aligned}
S_{x}= & \frac{4 \pi H^{2}}{\ell_{p}^{3}}\left[\frac{1}{r_{m}^{2}} S_{1}+a^{2} S_{2}+a^{4} r_{m}^{2} S_{3}+a^{4} r_{m}^{2} S_{4} \log \left(a r_{m}\right)-\frac{1}{2 r_{m}^{2}}+\frac{1}{12} a^{2} \log \left(r_{m}\right)\right] \\
= & \frac{4 \pi H^{2}}{\ell_{p}^{3}}\left[-\left(0.0073+0.0294 \gamma^{2}\right) a^{4} r_{m}^{2}+0.0085\left(1+3 \gamma^{2}\right) a^{4} r_{m}^{2} \log \left(a r_{m}\right)\right. \\
& \left.+0.0351 a^{2}-\frac{0.2156}{r_{m}^{2}}+\frac{a^{2}}{12} \log \left(r_{m}\right)\right]
\end{aligned}
$$

where we dropped the $\delta$ dependent divergent parts which are independent of $r_{m}$ and $\ell$ so do not contribute to the $c$-function. Notice that the last term is a universal term as it appears in the logarithmic part of (A4).

Similarly, we can compute the width of strip $\ell$ as a function of $r_{m}$. Start with

$$
\ell=2 \int_{0}^{\ell / 2} d x=2 \int_{0}^{r_{m}} \frac{d r}{|\dot{r}|}=2 \int_{0}^{r_{m}} d r \frac{1}{\sqrt{e^{2\left(B(r)-B\left(r_{m}\right)\right)}-1}}
$$

it follows then

$$
\begin{aligned}
\ell & =2 r_{m}\left(L_{1}+a^{2} r_{m}^{2} L_{2}+a^{4} r_{m}^{4} L_{3}+a^{4} r_{m}^{4} \log \left(a r_{m}\right) L_{4}\right) \\
& =2 r_{m}\left(0.4312+0.0158 a^{2} r_{m}^{2}-\left(0.0020+0.0062 \gamma^{2}\right) a^{4} r_{m}^{4}+0.0027\left(1+3 \gamma^{2}\right) a^{4} r_{m}^{4} \log \left(a r_{m}\right)\right)
\end{aligned}
$$

where $L_{i}$ 's are as follows 


$$
\begin{aligned}
L_{1}= & \int_{0}^{1} d u\left(\frac{1}{\sqrt{\frac{1}{u^{6}}-1}}\right)=0.4312 \\
L_{2}= & \int_{0}^{1} d u\left(\frac{1}{12 \sqrt{\frac{1}{u^{6}}-1}\left(u^{4}+u^{2}+1\right)}\right)=0.0158 \\
L_{3}= & \int_{0}^{1} d u\left(\frac{-163 u^{8}-1063 u^{6}+838 u^{2}+540\left(u^{8}+u^{6}+u^{4}\right) \log (u)+388}{64800 \sqrt{\frac{1}{u^{6}}-1}\left(u^{2}-1\right)\left(u^{4}+u^{2}+1\right)^{2}}\right. \\
& \left.+\frac{\gamma^{2}\left(-19 u^{4}+20 u^{4} \log (u)+19\right)}{800 \sqrt{\frac{1}{u^{6}}-1}\left(u^{6}-1\right)}\right) \\
= & -0.0020-0.0062 \gamma^{2} \\
L_{4}= & \int_{0}^{1} d u\left(\frac{\left(1+u^{2}\right)\left(1+3 \gamma^{2}\right)}{120 \sqrt{\frac{1}{u^{6}}-1}\left(u^{4}+u^{2}+1\right)}\right)=0.0027\left(1+3 \gamma^{2}\right) .
\end{aligned}
$$

Now we solve (A7) perturbatively to find $r_{m}$ in terms of $\ell$,

$$
\begin{aligned}
r_{m}= & 1.1596 \ell-0.0571 a^{2} \ell^{3}+\left(0.0162+0.0185 \gamma^{2}-0.0175 \gamma^{4}\right) a^{4} \ell^{5} \\
& -\left(0.0012+0.0070 \gamma^{2}+0.0105 \gamma^{4}\right) a^{4} \ell^{5} \log (a \ell) .
\end{aligned}
$$

Plugging in (A5), we obtain,

$$
\begin{aligned}
S_{x}= & \frac{4 \pi H^{2}}{\ell_{p}^{3}}\left[-\frac{0.1603}{\ell^{2}}+\frac{a^{2}}{12} \log \ell+0.0316 a^{2}-\left(0.0088+0.0293 \gamma^{2}+0.0048 \gamma^{4}\right) a^{4} \ell^{2}\right. \\
& \left.+\left(0.0079+0.0127 \gamma^{2}-0.0326 \gamma^{4}\right) a^{4} \ell^{2} \log (a \ell)\right] .
\end{aligned}
$$

Here we can find $c_{x}$ from (2.4) up to some constant,

$$
\begin{aligned}
c_{x} \sim & \ell^{3} \frac{\partial S_{x}}{\partial \ell} \sim 0.3207+0.0833 a^{2} \ell^{2}-\left(0.0098+0.0459 \gamma^{2}+0.0423 \gamma^{4}\right) a^{4} \ell^{4} \\
& +\left(0.0157+0.0253 \gamma^{2}-0.0653 \gamma^{4}\right) a^{4} \ell^{4} \log (a \ell)
\end{aligned}
$$

and its derivative

$$
\begin{aligned}
\frac{\partial c_{x}}{\partial \ell} \sim & 0.1667 a^{2} \ell-\left(0.0236+0.1584 \gamma^{2}+0.2345 \gamma^{4}\right) a^{4} \ell^{3} \\
& +\left(0.0628+0.1015 \gamma^{2}-0.2611 \gamma^{4}\right) a^{4} \ell^{3} \log (a \ell)
\end{aligned}
$$

Similar calculations can be done for the region along $y$-direction.

$$
\begin{aligned}
& S_{y}=\frac{4 \pi H^{2}}{\ell_{p}^{3}} r_{m} \int_{0}^{1} d u\left[\frac{e^{2 B\left(r_{m} u\right)-B\left(r_{m}\right)}}{e^{-h\left(r_{m} u\right)} \sqrt{e^{2 B\left(r_{m} u\right)-2 B\left(r_{m}\right)}-1}}-\left(\frac{1}{r_{m}^{3} u^{3}}-\frac{a^{2}}{24 r_{m} u}\right)\right] \\
& +\frac{4 \pi H^{2}}{\ell_{p}^{3}} r_{m} \int_{\delta / r_{m}}^{1} d u\left(\frac{1}{r_{m}^{3} u^{3}}-\frac{a^{2}}{24 r_{m} u}\right) \text {. }
\end{aligned}
$$


We write the first integral as

$$
\frac{4 \pi H^{2}}{\ell_{p}^{3}} r_{m}\left[\frac{1}{r_{m}^{3}} \tilde{S}_{1}+\frac{a^{2}}{r_{m}} \tilde{S}_{2}+a^{4} r_{m} \tilde{S}_{3}+a^{4} r_{m} \tilde{S}_{4} \log \left(a r_{m}\right)\right]
$$

with,

$$
\begin{aligned}
\tilde{S}_{1}= & \int_{0}^{1} d u\left[\frac{1}{\sqrt{\frac{1}{u^{6}}-1} u^{6}}-\frac{1}{u^{3}}\right]=0.2844 \\
\tilde{S}_{2}= & \int_{0}^{1} d u\left[\frac{u^{4}-u^{2}-1}{24 \sqrt{\frac{1}{u^{6}}-1} u^{4}\left(u^{4}+u^{2}+1\right)}+\frac{1}{24 u}\right]=0.0062 \\
\tilde{S}_{3}= & \int_{0}^{1} d u\left[\frac{-1579 u^{10}-2479 u^{8}+873 u^{6}+4279 u^{4}-221 u^{2}-873}{259200 \sqrt{\frac{1}{u^{6}}-1}\left(u^{2}-1\right)\left(u^{5}+u^{3}+u\right)^{2}}\right. \\
& -\frac{1080\left(u^{10}+u^{8}+u^{6}-3 u^{4}-3 u^{2}-3\right) \log (u)}{259200 \sqrt{\frac{1}{u^{6}}-1}\left(u^{2}-1\right)\left(u^{5}+u^{3}+u\right)^{2}} \\
& \left.-\frac{\gamma^{2}\left(13 u^{6}+10\left(u^{6}-3\right) \log (u)-19 u^{2}+6\right)}{800 \sqrt{\frac{1}{u^{6}}-1} u^{2}\left(u^{6}-1\right)}\right]=0.0033+0.0089 \gamma^{2} \\
\tilde{S}_{4}= & \int_{0}^{1} d u\left[\frac{-\left(u^{5}+u^{3}+3 u\right)\left(1+3 \gamma^{2}\right)}{240\left(u^{4}+u^{2}+1\right) \sqrt{1-u^{6}}}\right]=-0.0061\left(1+3 \gamma^{2}\right)
\end{aligned}
$$

The second integral in (A13) is found to be

$$
\frac{4 \pi H^{2}}{\ell_{p}^{3}} r_{m} \int_{\delta / r_{m}}^{1} d u\left(\frac{1}{r_{m}^{3} u^{3}}-\frac{a^{2}}{24 r_{m} u}\right)=\frac{4 \pi H^{2}}{\ell_{p}^{3}}\left(\frac{1}{24} a^{2} \log \left(\frac{\delta}{r_{m}}\right)+\frac{1}{2 \delta^{2}}-\frac{1}{2 r_{m}^{2}}\right)
$$

Then one finds

$$
\begin{aligned}
S_{y}= & \frac{4 \pi H^{2}}{\ell_{p}^{3}}\left[\frac{1}{r_{m}^{2}} \tilde{S}_{1}+a^{2} \tilde{S}_{2}+a^{4} r_{m}^{2} \tilde{S}_{3}+a^{4} r_{m}^{2} \tilde{S}_{4} \log \left(a r_{m}\right)-\frac{1}{2 r_{m}^{2}}-\frac{1}{24} a^{2} \log \left(r_{m}\right)\right] \\
= & \frac{4 \pi H^{2}}{\ell_{p}^{3}}\left[\left(0.0033+0.0089 \gamma^{2}\right) a^{4} r_{m}^{2}-0.0061\left(1+3 \gamma^{2}\right) a^{4} r_{m}^{2} \log \left(a r_{m}\right)+0.0062 a^{2}\right. \\
& \left.-\frac{0.2156}{r_{m}^{2}}-\frac{a^{2}}{24} \log \left(r_{m}\right)\right]
\end{aligned}
$$

where we again dropped the $\delta$ dependent divergent parts.

We then compute the width of strip $\ell$ as a function of $r_{m}$ as follows,

$$
\begin{aligned}
\ell & =2 \int_{0}^{\ell / 2} d y=2 \int_{0}^{r_{m}} \frac{d r}{|\dot{r}|}=2 \int_{0}^{r_{m}} d r \frac{1}{e^{-h \sqrt{e^{2 B(r)-2 B\left(r_{m}\right)}-1}}} \\
& =2 r_{m}\left(\tilde{L}_{1}+a^{2} r_{m}^{2} \tilde{L}_{2}+a^{4} r_{m}^{4} \tilde{L}_{3}+a^{4} r_{m}^{4} \log \left(a r_{m}\right) \tilde{L}_{4}\right) \\
& =\left(0.0051+0.0077 \gamma^{2}\right) a^{4} r_{m}^{5}-\left(0.0063+0.0189 \gamma^{2}\right) a^{4} r_{m}^{5} \log \left(a r_{m}\right)-0.0517 a^{2} r_{m}^{3}+0.8624 r_{m}
\end{aligned}
$$

where $\tilde{L}_{i}$ 's are given as 


$$
\begin{aligned}
\tilde{L}_{1}= & \int_{0}^{1} d u\left(\frac{1}{\sqrt{\frac{1}{u^{6}}-1}}\right)=0.4312 \\
\tilde{L}_{2}= & \int_{0}^{1} d u \frac{-3 u^{6}-3 u^{4}-3 u^{2}+2}{24 \sqrt{\frac{1}{u^{6}}-1}\left(u^{4}+u^{2}+1\right)}=-0.0259 \\
\tilde{L}_{3}= & \int_{0}^{1} d u\left[\frac{1552+6052 u^{2}-5125 u^{4}-9377 u^{6}-8477 u^{8}+5125 u^{10}+5125 u^{12}+5125 u^{14}}{259200 \sqrt{\frac{1}{u^{6}}-1}\left(u^{2}-1\right)\left(u^{4}+u^{2}+1\right)^{2}}\right. \\
& -\frac{1080 u^{4}\left(5 u^{10}+5 u^{8}+5 u^{6}-7 u^{4}-7 u^{2}-7\right) \log (u)}{259200 \sqrt{\frac{1}{u^{6}}-1}\left(u^{2}-1\right)\left(u^{4}+u^{2}+1\right)^{2}} \\
& \left.+\frac{\gamma^{2}\left(25 u^{10}-44 u^{4}+10\left(7-5 u^{6}\right) u^{4} \log (u)+19\right)}{800 \sqrt{\frac{1}{u^{6}}-1}\left(u^{6}-1\right)}\right] \\
\tilde{L}_{4}= & \int_{0}^{1} d u \frac{\left(-5 u^{8}-5 u^{6}-5 u^{4}+2 u^{2}+2\right)\left(1+3 \gamma^{2}\right)}{240 \sqrt{\frac{1}{u^{6}}-1}\left(u^{4}+u^{2}+1\right)}=-0.00314521\left(1+3 \gamma^{2}\right) \\
& 0.0026+0.0038 \gamma^{2} \\
& \\
&
\end{aligned}
$$

Now we solve (A18) perturbatively to find $r_{m}$ in terms of $\ell$,

$$
r_{m}=1.1596 \ell+0.0935 a^{2} \ell^{3}+\left(0.0124-0.0119 \gamma^{2}\right) a^{4} \ell^{5}+\left(0.0153+0.0459 \gamma^{2}\right) a^{4} \ell^{5} \log (a \ell) .
$$

Plugging in (A17), we obtain,

$$
\begin{aligned}
S_{y}= & \frac{4 \pi H^{2}}{\ell_{p}^{3}}\left[-\frac{0.1603}{\ell^{2}}-\frac{a^{2}}{24} \log (\ell)+0.0259 a^{2}+\left(0.0002+0.0050 \gamma^{2}\right) a^{4} \ell^{2}\right. \\
& \left.-\left(0.0039+0.0118 \gamma^{2}\right) a^{4} \ell^{2} \log (a \ell)\right] .
\end{aligned}
$$

Then the $c$-function and its derivative can be found as

$$
\begin{aligned}
c_{y} & \sim \ell^{3} \frac{\partial S_{y}}{\partial \ell} \sim 0.3207-0.0417 a^{2} \ell^{2}-\left(0.0034+0.0018 \gamma^{2}\right) a^{4} \ell^{4}-\left(0.0079+0.0236 \gamma^{2}\right) a^{4} \ell^{4} \log (a \ell) \\
\frac{\partial c_{y}}{\partial \ell} & \sim-0.0833 a^{2} \ell-\left(0.0216+0.0306 \gamma^{2}\right) a^{4} \ell^{3}-\left(0.0314+0.0943 \gamma^{2}\right) a^{4} \ell^{3} \log (a \ell) .
\end{aligned}
$$

\section{APPENDIX B: THE RG-FLOW EQUATION}

In this Appendix, we present the details of deriving the RG-flow given in (3.4). First, we find variation of $S$ with respect to $\ell$, reminding that it has an explicit $\ell$ dependence through the integral upper bound and an implicit one through $r(x, \ell)$. One finds,

$\frac{d S}{d \ell}=\left.\frac{4 \pi H^{d-1}}{\ell_{p}^{d}} \frac{e^{(d-1) A+h}}{\sqrt{\dot{r}^{2}+1}}\left[\frac{1}{2}\left(1-\frac{d \epsilon}{d \ell}\right)\left(\dot{r}^{2}+1\right)+\dot{r} \frac{\partial r}{\partial \ell}\right]\right|_{x=\frac{\ell-\epsilon}{2}}$, where we have used (2.16) and integration by parts. Following [18], we take a variation of $r\left(\frac{\ell-\epsilon}{2}, \ell\right)=r_{c}$ with respect to $\ell$ to find

$$
\left[\frac{\dot{r}(x, \ell)}{2}\left(1-\frac{d \epsilon}{d \ell}\right)+\frac{\partial r(x, \ell)}{\partial \ell}\right]_{x=\frac{\ell-\epsilon}{2}}=0 .
$$

Plugging in (B1), we find

$$
\frac{d S}{d \ell}=-\left.\frac{4 \pi H^{d-1}}{\ell_{p}^{d}} \frac{1}{K_{d}(\ell)} \frac{1}{\dot{r}} \frac{\partial r}{\partial \ell}\right|_{x=\frac{\ell-\epsilon}{2}} .
$$


In the UV regime, $r \rightarrow 0$, the background is expected to be $A d S$, so we have,

$$
\begin{aligned}
& A(r) \sim-\log (r) \\
& h(r) \sim 0
\end{aligned}
$$

Then we solve (2.17) to get $\dot{r}=-\sqrt{K_{d}^{2} r^{2-2 d}-1}$ and integrate to find $x$,

$$
x-\frac{\ell}{2}=\frac{1}{d-1} K_{d}^{\frac{1}{d-1}} F\left(K_{d} r^{1-d}\right)
$$

where

$$
F(u)=\int \frac{u^{\frac{d}{1-d}}}{\sqrt{u^{2}-1}} d u .
$$

It is clear that at the boundary $F(u \rightarrow \infty)=0$. Now following [18], take a partial derivative of (B5) with respect to $\ell$, and solve for $\partial r / \partial \ell$,

$$
\begin{aligned}
\frac{\partial r}{\partial \ell}= & \frac{1}{(d-1)} \frac{K_{d}^{\prime}(\ell)}{K_{d}} r+\frac{1}{2(d-1)^{2}} K_{d}^{\frac{2}{d-1}} \sqrt{K_{d}^{2} r^{2-2 d}-1} \\
& \times\left((d-1)^{2}-2 K_{d}^{\frac{d}{1-d}} K_{d}^{\prime}(\ell) F(u)\right)
\end{aligned}
$$

then divide by $\dot{r}=-\sqrt{K_{d}^{2} r^{2-2 d}-1}$ and take the limit $r \rightarrow 0$ to find,

$$
\left.\frac{1}{\dot{u}} \frac{\partial u}{\partial \ell}\right|_{x=\ell}=-\frac{1}{2}
$$

It follows then

$$
\frac{d S}{d \ell}=\frac{2 \pi H^{d-1}}{\ell_{p}^{d}} \frac{1}{K_{d}}
$$

which is the Eq. (3.2).

Now our task is to find the flow $d c_{d} / d r_{m}$ where $r_{m}$ is defined as $r_{m}=r(0, \ell)$ with $\dot{r}(0, \ell)=0$. We proceed as

$$
\frac{d c_{d}}{d r_{m}}=\beta_{d} \frac{2 \pi d \ell^{d-1}}{\ell_{p}^{d} K_{d}}\left(\frac{d \ell}{d r_{m}}+\frac{1}{d} B^{\prime}\left(r_{m}\right) \ell\right) .
$$

On the other hand, $\ell$ can be found as a function of $r_{m}$,

$\ell=2 \int_{0}^{\ell / 2} d x=-2 \int_{0}^{r_{m}} \frac{d r}{\dot{r}}=2 \int_{0}^{r_{m}} d r \frac{1}{\sqrt{K_{d}^{2} e^{2 B}-1}}$
Now define $w=K_{d} e^{B}$ and

$$
G(w):=\int \frac{w^{-\left(1+\frac{1}{d}\right)}}{\sqrt{w^{2}-1}} d w
$$

which is indeed a hypergeometric function with $G(w \rightarrow$ $\infty)=0$ at the boundary $\rightarrow 0$. Then we have

$$
\begin{aligned}
\ell & =2 \int_{r=0}^{r=r_{m}} \frac{K_{d}^{1 / d} e^{B / d}}{B^{\prime}} d G \\
& =2 \frac{G(1)}{B^{\prime}\left(r_{m}\right)}-2 \int_{0}^{r_{m}} K_{d}^{1 / d} G\left(\frac{e^{B / d}}{B^{\prime}}\right)^{\prime} d r
\end{aligned}
$$

where the first term is the boundary term. We now take a derivative with respect to $r_{m}$,

$$
\begin{aligned}
\frac{d \ell}{d r_{m}}= & -\frac{2}{d} G(1)+\frac{2}{d} B^{\prime}\left(r_{m}\right) \int_{0}^{r_{m}} K_{d}^{1 / d} G\left(\frac{e^{B / d}}{B^{\prime}}\right)^{\prime} d r \\
& -2 \int_{0}^{r_{m}} K_{d}^{1 / d} \frac{d G}{d r_{m}}\left(\frac{e^{B / d}}{B^{\prime}}\right)^{\prime} d r \\
= & -\frac{2}{d} G(1)+\frac{2}{d} B^{\prime}\left(r_{m}\right) \int_{0}^{r_{m}}\left(K_{d}^{1 / d} G(w)+\frac{d e^{-B / d}}{\sqrt{w^{2}-1}}\right) \\
& \times\left(\frac{e^{B / d}}{B^{\prime}}\right)^{\prime} d r
\end{aligned}
$$

in which we have used

$$
\frac{d G}{d r_{m}}=-e^{B} K_{d} B^{\prime}\left(r_{m}\right) \frac{d G}{d w} .
$$

Now plugging (B13) and (B14) in (B10) we obtain,

$$
\begin{aligned}
\frac{d c_{d}}{d r_{m}} & =\beta_{d} \frac{4 \pi d \ell^{d-1}}{\ell_{p}^{d}} B^{\prime}\left(r_{m}\right) \int_{0}^{r_{m}} \frac{e^{-B / d}}{\sqrt{K_{d}^{2} e^{2 B}-1}}\left(\frac{e^{B / d}}{B^{\prime}}\right)^{\prime} d r \\
& =\beta_{d} \frac{4 \pi d \ell^{d-1}}{\ell_{p}^{d}} B^{\prime}\left(r_{m}\right) \int_{0}^{\ell / 2} d x e^{-B / d}\left(\frac{e^{B / d}}{B^{\prime}}\right)^{\prime} \\
& =\beta_{d} \frac{4 \pi \ell^{d-1}}{\ell_{p}^{d}} B^{\prime}\left(r_{m}\right) \int_{0}^{\ell / 2} d x \frac{B^{\prime 2}-d B^{\prime \prime}}{B^{\prime 2}} .
\end{aligned}
$$


[1] A. B. Zamolodchikov, Irreversibility of the flux of the renormalization group in a $2 \mathrm{D}$ field theory, Pis'ma $\mathrm{Zh}$. Eksp. Teor. Fiz. 43, 565 (1986) [JETP Lett. 43, 730 (1986)].

[2] P. Calabrese and J. L. Cardy, Entanglement entropy and quantum field theory, J. Stat. Mech. (2004) P06002.

[3] P. Calabrese and J. Cardy, Entanglement entropy and conformal field theory, J. Phys. A 42, 504005 (2009).

[4] H. Casini and M. Huerta, A finite entanglement entropy and the c-theorem, Phys. Lett. B 600, 142 (2004).

[5] H. Casini and M. Huerta, A c-theorem for the entanglement entropy, J. Phys. A 40, 7031 (2007).

[6] J. L. Cardy, Is there a c theorem in four-dimensions?, Phys. Lett. B 215, 749 (1988).

[7] Z. Komargodski and A. Schwimmer, On renormalization group flows in four dimensions, J. High Energy Phys. 12 (2011) 099.

[8] Z. Komargodski, The constraints of conformal symmetry on RG flows, J. High Energy Phys. 07 (2012) 069.

[9] H. Casini and M. Huerta, On the RG running of the entanglement entropy of a circle, Phys. Rev. D 85, 125016 (2012).

[10] D. Z. Freedman, S. S. Gubser, K. Pilch, and N. P. Warner, Renormalization group flows from holography supersymmetry and a c theorem, Adv. Theor. Math. Phys. 3, 363 (1999).

[11] L. Girardello, M. Petrini, M. Porrati, and A. Zaffaroni, Novel local CFT and exact results on perturbations of $N=4$ superYang Mills from AdS dynamics, J. High Energy Phys. 12 (1998) 022.

[12] L. Girardello, M. Petrini, M. Porrati, and A. Zaffaroni, The supergravity dual of $N=1$ superYang-Mills theory, Nucl. Phys. B569, 451 (2000).

[13] R. C. Myers and A. Sinha, Seeing a c-theorem with holography, Phys. Rev. D 82, 046006 (2010).

[14] R. C. Myers and A. Sinha, Holographic c-theorems in arbitrary dimensions, J. High Energy Phys. 01 (2011) 125.

[15] S. Ryu and T. Takayanagi, Holographic Derivation of Entanglement Entropy from AdS/CFT, Phys. Rev. Lett. 96, 181602 (2006).

[16] S. Ryu and T. Takayanagi, Aspects of holographic entanglement entropy, J. High Energy Phys. 08 (2006) 045.

[17] T. Nishioka, Entanglement entropy: Holography and renormalization group, Rev. Mod. Phys. 90, 035007 (2018).

[18] R. C. Myers and A. Singh, Comments on holographic entanglement entropy and RG flows, J. High Energy Phys. 04 (2012) 122.

[19] B. Swingle, Entanglement does not generally decrease under renormalization, J. Stat. Mech. (2014) P10041.

[20] S. Cremonini and X. Dong, Constraints on renormalization group flows from holographic entanglement entropy, Phys. Rev. D 89, 065041 (2014).
[21] D. Giataganas, U. Grsoy, and J.F. Pedraza, StronglyCoupled Anisotropic Gauge Theories and Holography, Phys. Rev. Lett. 121, 121601 (2018).

[22] D. Giataganas, D. S. Lee, and C. P. Yeh, Quantum fluctuation and dissipation in holographic theories: A unifying study scheme, J. High Energy Phys. 08 (2018) 110.

[23] D. Giataganas, Stochastic motion of heavy quarks in holography: A theory-independent treatment, Proc. Sci. CORFU2017 (2018) 032 [arXiv:1805.09011].

[24] S. Jain, R. Samanta, and S. P. Trivedi, The shear viscosity in anisotropic phases, J. High Energy Phys. 10 (2015) 028.

[25] D. Giataganas, Observables in strongly coupled anisotropic theories, Proc. Sci. Corfu2012 (2013) 122 [arXiv:1306.1404].

[26] D. Giataganas, Probing strongly coupled anisotropic plasma, J. High Energy Phys. 07 (2012) 031.

[27] U. Grsoy, M. Jrvinen, G. Nijs, and J. F. Pedraza, Inverse anisotropic catalysis in holographic QCD, J. High Energy Phys. 04 (2019) 071.

[28] S. S. Gubser and A. Nellore, Mimicking the QCD equation of state with a dual black hole, Phys. Rev. D 78, 086007 (2008).

[29] S. S. Gubser, A. Nellore, S. S. Pufu, and F. D. Rocha, Thermodynamics and Bulk Viscosity of Approximate Black Hole Duals to Finite Temperature Quantum Chromodynamics, Phys. Rev. Lett. 101, 131601 (2008).

[30] M. Ghasemi and S. Parvizi, Curved corner contribution to the entanglement entropy in an anisotropic spacetime, arXiv:1905.01675.

[31] M. Alishahiha and H. Yavartanoo, On holography with hyperscaling violation, J. High Energy Phys. 11 (2012) 034.

[32] S. Pal and B. Grinstein, Weyl consistency conditions in nonrelativistic quantum field theory, J. High Energy Phys. 12 (2016) 012.

[33] I. Arav, I. Hason, and Y. Oz, Spontaneous breaking of nonrelativistic scale symmetry, J. High Energy Phys. 10 (2017) 063.

[34] R. Auzzi, S. Baiguera, F. Filippini, and G. Nardelli, On Newton-Cartan local renormalization group and anomalies, J. High Energy Phys. 11 (2016) 163.

[35] S. N. Solodukhin, The a-theorem and entanglement entropy, arXiv:1304.4411.

[36] J. K. Ghosh, E. Kiritsis, F. Nitti, and L. T. Witkowski, Holographic RG flows on curved manifolds and the $F$-theorem, J. High Energy Phys. 02 (2019) 055.

[37] P. Liu, C. Niu, and J. P. Wu, The effect of anisotropy on holographic entanglement entropy and mutual information, Phys. Lett. B 796, 155 (2019).

[38] C. S. Chu and D. Giataganas, $c$-theorem for anisotropic RG flows from holographic entanglement entropy, Phys. Rev. D 101, 046007 (2020). 